\title{
Network Coding Capacity with a Constrained Number of Coding Nodes
}

\author{
Jillian Cannons and Kenneth Zeger
}

\begin{abstract}
We study network coding capacity under a constraint on the total number of network nodes that can perform coding. That is, only a certain number of network nodes can produce coded outputs, whereas the remaining nodes are limited to performing routing. We prove that every non-negative, monotonically non-decreasing, eventually constant, rationalvalued function on the non-negative integers is equal to the capacity as a function of the number of allowable coding nodes of some directed acyclic network.
\end{abstract}

\section{INTRODUCTION}

Let $\mathbb{N}$ denote the positive integers, and let $\mathbb{R}$ and $\mathbb{Q}$ denote the real and rational numbers, respectively, with a superscript "+" denoting restriction to positive values. In this paper, a network is a directed acyclic multigraph $G=(V, E)$, some of whose nodes are information sources or receivers (e.g. see [11]). Associated with the sources are $m$ generated messages, where the $i^{\text {th }}$ source message is assumed to be a vector of $k_{i}$ arbitrary elements of a fixed finite alphabet, $\mathcal{A}$, of size at least 2. At any node in the network, each out-edge carries a vector of $n$ alphabet symbols which is a function (called an edge function) of the vectors of symbols carried on the in-edges to the node, and of the node's message vectors if it is a source. Associated with each receiver are demands, which are subsets of the network messages. Each receiver has decoding functions which map its inputs to vectors of symbols in an attempt to produce the messages demanded at the receiver. The goal is for each receiver to deduce its demanded messages from its in-edges and source messages.

A $\left(k_{1}, \ldots, k_{m}, n\right)$ fractional code is a collection of edge functions, one for each edge in the network, and decoding functions, one for each demand of each node in the network. A $\left(k_{1}, \ldots, k_{m}, n\right)$ fractional solution is a $\left(k_{1}, \ldots, k_{m}, n\right)$ fractional code which results in every receiver being able to compute its demands via its decoding functions, for all possible assignments of length- $k_{i}$ vectors over the alphabet to the $i^{t h}$ source message, for all $i$.

For each $i$, the ratio $k_{i} / n$ can be thought of as the rate at which source $i$ injects data into the network. If a network has a $\left(k_{1}, \ldots, k_{m}, n\right)$ fractional solution over some alphabet, then we say that $\left(k_{1} / n, \ldots, k_{m} / n\right)$ is an achievable rate vector, and we define the achievable rate region of the network as the $\operatorname{set}^{1}$

$$
S=\left\{r \in \mathbb{Q}^{m}: r \text { is an achievable rate vector }\right\} .
$$

This work was supported by the National Science Foundation and the UCSD Center for Wireless Communications

The authors are with the Department of Electrical and Computer Engineering, University of California, San Diego, La Jolla, CA 92093-0407 (jcannons@ucsd.edu, zeger@ucsd.edu)

${ }^{1}$ Alternatively, sometimes the closure $\bar{S}$, with respect to $\mathbb{R}^{m}$, is taken as the definition of the achievable rate region.
We define the uniform coding capacity of a network to be

$$
\mathcal{C}^{\text {uniform }}=\sup \left\{\min _{1 \leq i \leq m} r_{i}:\left(r_{1}, \ldots, r_{m}\right) \in S\right\} .
$$

We define the average coding capacity of a network to be

$$
\mathcal{C}^{\text {average }}=\sup \left\{\frac{1}{m} \sum_{i=1}^{m} r_{i}:\left(r_{1}, \ldots, r_{m}\right) \in S\right\} .
$$

Routing nodes have edge functions that simply copy specified input components to output components. ${ }^{2}$ If a network's edge functions are restricted to routing functions, then $\mathcal{C}^{\text {average }}$ and $\mathcal{C}^{\text {uniform }}$ will be referred to as the average routing capacity and uniform routing capacity, respectively.

Ahlswede, Cai, Li, and Yeung [1] exhibited a network whose average and uniform coding capacities are equal and are larger than its routing capacity. $\mathrm{Li}$, Yeung, and Cai [7] showed in the special case of a multicast network, the average and uniform coding capacities are both equal to the linear coding capacity. It was shown in [3], that for all networks, the uniform coding capacity is independent of the alphabet size (only slight modification of the proof is required for the average coding capacity case). Clearly the average and uniform routing capacities are also independent of the alphabet size. It is also known that the coding capacity might not be achievable [4].

In terms of the coding gain of a network, Sanders, Egner, and Tolhuizen [8] showed that for directed networks, the throughput achievable with network coding can be arbitrarily larger than that achievable with only routing. An important problem is to determine how many nodes in a network are required to perform coding in order for the network to achieve its coding capacity (or to achieve a coding rate arbitrarily close to its capacity if the capacity is not actually achievable). A network node is said to be a coding node if at least one of its out-edges has a non-routing edge function. A similar problem is to determine the number of coding nodes needed to assure the network has a solution (i.e. a $\left(k_{1}, \ldots, k_{m}, n\right)$ fractional solution with $k_{1}=\cdots=k_{m}=$ $n=1)$. The number of required coding nodes in both problems can in general range anywhere from zero up to the total number of nodes in the network.

For the special case of multicast networks, the problem of finding a minimal set of coding nodes to solve a network has been examined previously in [2], [5], [6], [9], the results of which are summarized as follows. Langberg, Sprintson, and

\footnotetext{
${ }^{2}$ If an edge function for an out-edge of a node depends only on the symbols of a single in-edge of that node, then, without loss of generality, we assume that the out-edge simply carries the same vector of symbols (i.e. routes the vector) as the in-edge it depends on
} 
Bruck [6] determined upper bounds on the minimum number of coding nodes required for a solution. Their bounds are given as functions of the number of messages and the number of receivers. Tavory, Feder, and Ron [9] showed that with 2 source messages, the minimum number of coding nodes required for a solution is independent of the total number of nodes in the network, while Fragouli and Soljanin [5] showed this minimum to be upper bounded by the number of receivers. Bhattad, Ratnakar, Koetter, and Narayanan [2] gave a method for finding solutions with reduced numbers of coding nodes, but their method may not find the minimum possible number of coding nodes. Wu, Jain, and Kung [10] demonstrated that only certain network edges require coding functions. This fact indirectly influences the number of coding nodes required, but does not immediately give an algorithm for finding a minimum node set.

We study here a related (and more general) problem, namely how network coding capacities can vary as functions of the number of allowable coding nodes. Our main result, given in Theorem 2.2, shows that the capacities of networks, as functions of the number of allowable coding nodes, can be almost anything. That is, the class of directed acyclic networks can witness arbitrary amounts of capacity gain by using arbitrarily sized node subsets for coding.

\section{Node-Limited Coding CAPACITY}

For each non-negative integer $i$, a $\left(k_{1}, \ldots, k_{m}, n\right)$ fractional $i$-node coding solution for a network is a $\left(k_{1}, \ldots, k_{m}, n\right)$ fractional coding solution with at most $i$ coding nodes (i.e. have output edges using non-routing edge functions). ${ }^{3}$ For each $i$, denote by $\mathcal{C}_{i}^{\text {average }}$ and $\mathcal{C}_{i}^{\text {uniform }}$ the average and uniform coding capacities, respectively, when solutions are restricted to those having at most $i$ coding nodes (we make the convention for all $i>|V|$, that $\mathcal{C}_{i}^{\text {average }}=$ $\mathcal{C}_{|V|}^{\text {average }}$ and $\left.\mathcal{C}_{i}^{\text {uniform }}=\mathcal{C}_{|V|}^{\text {uniform }}\right)$. We call $\mathcal{C}_{i}^{\text {average }}$ and $\mathcal{C}_{i}^{\text {uniform }}$ the node-limited average capacity function and node-limited uniform capacity function, respectively.

For a given number of coding nodes, if a network's nodelimited capacity is achievable, then it must be rational, and cannot decrease if more nodes are allowed to perform coding (since one can always choose not to use extra nodes for coding). By examining the admissible forms of $\mathcal{C}_{i}^{\text {average }}$ and $\mathcal{C}_{i}^{\text {uniform }}$ we gain insight into the possible capacity benefits of performing network coding at a limited number of nodes.

Theorem 2.2 demonstrates that the node-limited capacities of networks can vary more-or-less arbitrarily as functions of the number of allowable coding nodes.

Lemma 2.1: For any positive integers $p$ and $q \geq p$, the network $\mathcal{N}(p, q)$ shown in Fig. 1 has node-limited average and uniform capacity functions given by

$$
\mathcal{C}_{i}^{\text {average }}=\mathcal{C}_{i}^{\text {uniform }}=\left\{\begin{array}{cl}
p / q & \text { for } i=0 \\
1 & \text { for } i \geq 1
\end{array}\right.
$$

\footnotetext{
${ }^{3}$ Arbitrary decoding is allowed at receiver nodes and receiver nodes only count towards the total count of coding nodes in a network if they have out-edges performing coding.
}

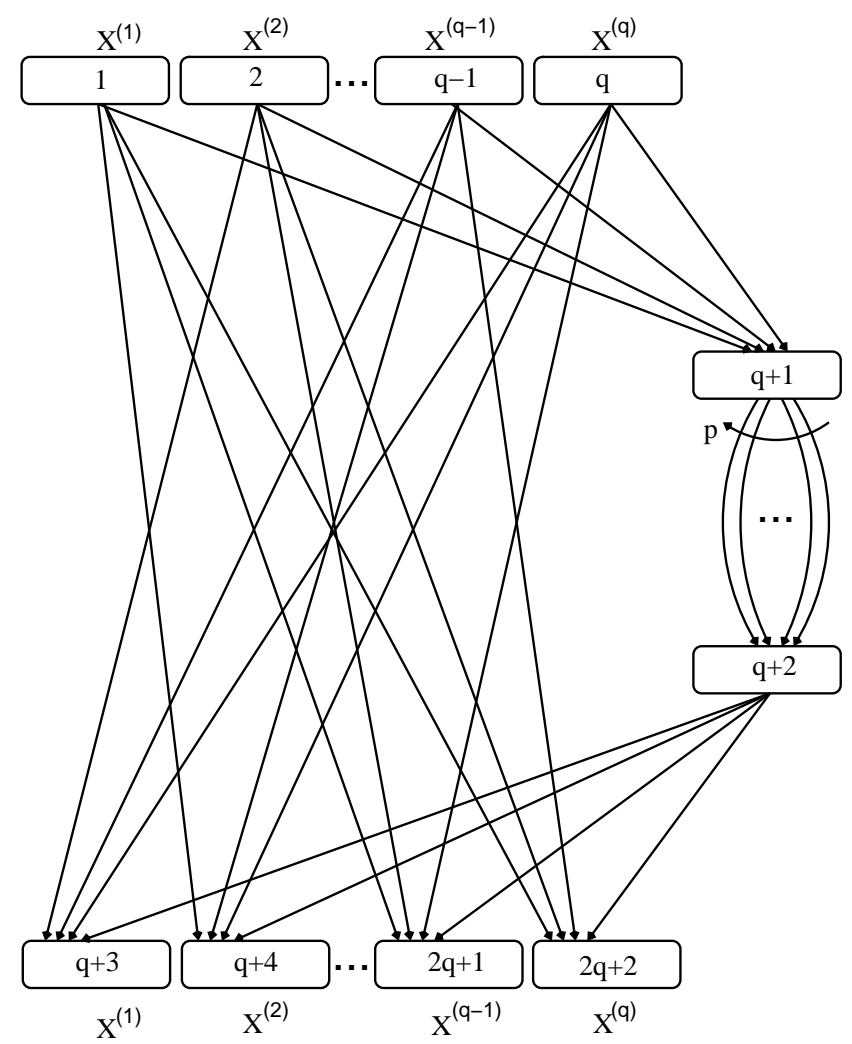

Fig. 1. The network $\mathcal{N}(p, q)$. Nodes $n_{1}, \ldots, n_{q}$ are the sources, with node $n_{i}$ providing message $X^{(i)}$, for $1 \leq i \leq q$. Nodes $n_{q+3}, \ldots, n_{2 q+2}$ are the receivers, with node $n_{i}$ demanding message $X^{(i-q-2)}$, for $q+3 \leq$ $i \leq 2 q+2$. Every source has one out-edge going to node $n_{q+1}$ and every receiver has one in-edge coming from node $n_{q+2}$. Also, every source $n_{i}$ has an out-edge going to receiver $n_{q+2+j}$, for all $j \neq i$. There are $p$ parallel edges from node $n_{q+1}$ to node $n_{q+2}$.

Theorem 2.2: Every monotonically non-decreasing, eventually constant function $f: \mathbb{N} \cup\{0\} \rightarrow \mathbb{Q}^{+}$is the nodelimited average and uniform capacity function of some directed acyclic network.

Proof:

Suppose $f: \mathbb{N} \cup\{0\} \rightarrow \mathbb{Q}^{+}$is given by

$$
f(i)= \begin{cases}p_{i} / q_{i} & \text { for } 0 \leq i<s \\ p_{s} / q_{s} & \text { for } i \geq s\end{cases}
$$

where $p_{0}, \ldots, p_{s}, q_{0}, \ldots, q_{s}$ are positive integers such that $p_{0} / q_{0} \leq p_{1} / q_{1} \leq \cdots \leq p_{s} / q_{s}$. Define the positive integers

$$
\begin{aligned}
b & =\operatorname{lcm}\left(p_{s} q_{i}: 0 \leq i<s\right) \\
a_{i} & =\frac{p_{i} / q_{i}}{p_{s} / q_{s}} \cdot b
\end{aligned}
$$

and construct network $\mathcal{N}$ as in Fig. 2, which has $m=b$ messages with $\mathcal{N}\left(a_{0}, b\right), \ldots, \mathcal{N}\left(a_{s-1}, b\right)$ as building blocks.

Let $\mathcal{C}_{i}^{\text {uniform }}$ and $\mathcal{C}_{i}^{\text {average }}$ denote the uniform and average node-limited capacity functions of network $\mathcal{N}$. Also, for $j=0, \ldots, s-1$, let $C_{j, i}^{\text {uniform }}$ and $C_{j, i}^{\text {average denote the }}$ uniform and average node-limited capacity functions of the sub-block $\mathcal{N}\left(a_{j}, b\right)$. There are exactly $s$ nodes in $\mathcal{N}$ that have 


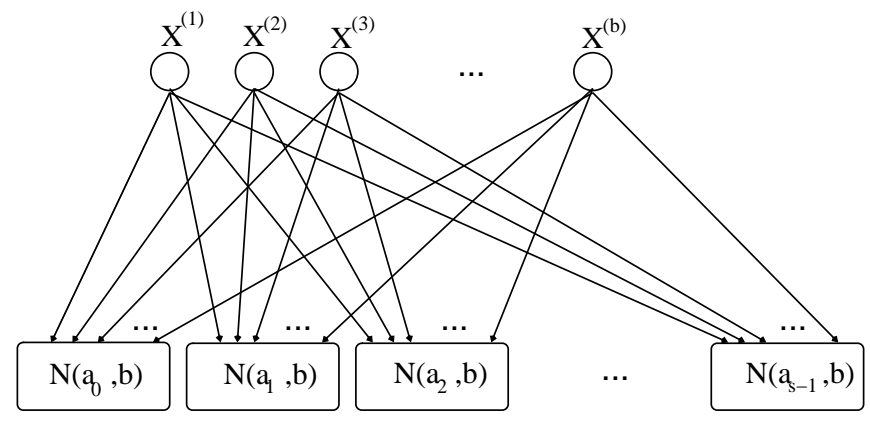

Fig. 2. The network $\mathcal{N}$ has $b$ source nodes, each emitting one message. Each source node has an out-edge to each sub-block $\mathcal{N}\left(a_{0}, b\right), \ldots, \mathcal{N}\left(a_{s-1}, b\right)$. Specifically, in each sub-block $\mathcal{N}\left(a_{i}, b\right)$, the previous source messages are removed, however each previous source node is connected by an in-edge from the unique corresponding source node in $\mathcal{N}$. Each sub-block $\mathcal{N}\left(a_{i}, b\right)$ has routing capacity $a_{i} / b=\left(p_{i} / q_{i}\right) /\left(p_{s} / q_{s}\right)$.

more than one in-edge, and which are therefore potential coding nodes (i.e. one potential coding node per block).

For each $i=0, \ldots, s-1$, in order to obtain a $\left(k_{1}, \ldots, k_{m}, n\right)$ fractional $i$-node coding solution, the quantity $\left(k_{1}+\cdots+k_{m}\right) /(m n)$ must be at most

$$
\min _{j} \frac{p_{j} / q_{j}}{p_{s} / q_{s}}
$$

where the minimization is taken over all $j$ for which subblock $\mathcal{N}\left(a_{j}, b\right)$ has no coding nodes (as per Lemma 2.1). That is, we must have

$$
\frac{k_{1}+\cdots+k_{m}}{m n} \leq \frac{p_{i} / q_{i}}{p_{s} / q_{s}} .
$$

Thus, the node-limited average and uniform coding capacities of $\mathcal{N}$ using $i$ coding nodes are at most the respective routing capacities of sub-block $\mathcal{N}\left(a_{i}, b\right)$ of $\mathcal{N}$, i.e.

$$
\begin{aligned}
\mathcal{C}_{i}^{\text {uniform }} \leq C_{i, 0}^{\text {uniform }}=a_{i} / b=\left(p_{i} / q_{i}\right) /\left(p_{s} / q_{s}\right) \\
\mathcal{C}_{i}^{\text {average }} \leq C_{i, 0}^{\text {average }}=a_{i} / b=\left(p_{i} / q_{i}\right) /\left(p_{s} / q_{s}\right) .
\end{aligned}
$$

These upper bounds are achievable by using coding only at the one possible coding node in each of the sub-blocks $\mathcal{N}\left(a_{0}, b\right), \ldots, \mathcal{N}\left(a_{i-1}, b\right)$. By taking

$$
\begin{aligned}
k_{1}=\cdots=k_{m} & =\operatorname{lcm}\left(a_{i}, \ldots, a_{s-1}\right) \\
n & =b \cdot \operatorname{lcm}\left(a_{i}, \ldots, a_{s-1}\right) / a_{i}
\end{aligned}
$$

we can obtain a $\left(k_{1}, \ldots, k_{m}, n\right)$ fractional $i$-node coding solution with coding nodes in sub-blocks $\mathcal{N}\left(a_{0}, b\right), \ldots, \mathcal{N}\left(a_{i-1}, b\right)$ and only routing edge-functions in sub-blocks $\mathcal{N}\left(a_{i}, b\right), \ldots, \mathcal{N}\left(a_{s-1}, b\right)$. With such a solution, the coding capacity $C_{j, 1}^{\text {uniform }}=C_{j, 1}^{\text {average }}=1$ is achieved in each sub-block $\mathcal{N}\left(a_{0}, b\right), \ldots, \mathcal{N}^{\prime}\left(a_{i-1}, b\right)$, and the (unchanging) routing capacity $C_{i, 0}^{\text {uniform }}=C_{i, 0}^{\text {average }}$ is achieved in each sub-block $\mathcal{N}\left(a_{i}, b\right), \ldots, \mathcal{N}\left(a_{s-1}, b\right)$.

Thus, network $\mathcal{N}$ has node-limited capacity functions

$\mathcal{C}_{i}^{\text {average }}=\mathcal{C}_{i}^{\text {uniform }}=\left\{\begin{array}{cl}\left(p_{i} / q_{i}\right) /\left(p_{s} / q_{s}\right) & \text { for } 0 \leq i<s \\ 1 & \text { for } i \geq s .\end{array}\right.$

If we replace each message of $\mathcal{N}$ by $q_{s}$ new independent messages and change the receiver demands accordingly, and if we replace each directed edge of $\mathcal{N}$ by $p_{s}$ parallel edges in the same orientation, then the resulting network $\hat{\mathcal{N}}$ will have node-limited average and uniform capacity functions

$$
\hat{\mathcal{C}}_{i}^{\text {average }}=\hat{\mathcal{C}}_{i}^{\text {uniform }}=\left(p_{s} / q_{s}\right) \mathcal{C}_{i}^{\text {uniform }}=f(i) .
$$

One consequence of Theorem 2.2 is that large coding gains can be suddenly obtained after an arbitrary number of nodes has been used for coding.

In Theorem 2.2 the existence of networks that achieve prescribed rational-valued node-limited capacity functions was established. It is known in general that not all networks necessarily achieve their capacities [4]. It is presently unknown, however, whether a network coding capacity could be irrational. Thus, we are not presently able to extend Theorem 2.2 to real-valued functions. Nevertheless, Theorem 2.2 does immediately imply the following asymptotic achievability result for real-value functions.

Corollary 2.3: Every monotonically non-decreasing, eventually constant function $f: \mathbb{N} \cup\{0\} \rightarrow \mathbb{R}^{+}$is the limit of the node-limited uniform (or average) capacity functions of some sequence of directed acyclic networks.

\section{REFERENCES}

[1] R. Ahlswede, N. Cai, S.-Y. R. Li, and R. W. Yeung, "Network information flow", IEEE Transactions on Information Theory, vol. 46, no. 4, pp. 1204 - 1216, July 2000.

[2] K. Bhattad, N. Ratnakar, R. Koetter, and K. R. Narayanan, "Minimal network coding for multicast", Proceedings of the 2005 IEEE International Symposium on Information Theory (ISIT), Adelaide, Australia, September 4 - 9, 2005.

[3] J. Cannons, R. Dougherty, C. Freiling, and K. Zeger, "Network routing capacity", IEEE Transactions on Information Theory, vol. 52, no. 3, pp. 777 - 788, March 2006.

[4] R. Dougherty, C. Freiling, and K. Zeger, "Unachievability of network coding capacity", IEEE Transactions on Information Theory \& IEEE/ACM Transactions on Networking (joint issue), vol. 52, no. 6 , pp. $2365-2372$, June 2006

[5] C. Fragouli and E. Soljanin, "Information flow decomposition for network coding", IEEE Transactions on Information Theory, vol. 52 , no. 3, pp. 829 - 848, March 2006.

[6] M. Langberg, A. Sprintson, and J. Bruck, "The encoding complexity of network coding", IEEE Transactions on Information Theory and IEEE/ACM Transactions on Networking (joint issue), vol. 52, no. 6, pp. 2386 - 2397, June 2006.

[7] S.-Y. R. Li, R. W. Yeung, and N. Cai, "Linear network coding", IEEE Transactions on Information Theory, vol. 49, no. 2, pp. 371 - 381, February 2003.

[8] P. Sanders, S. Egner, L. Tolhuizen, "Polynomial time algorithms for network information flow", 15th ACM Symposium on Parallelism in Algorithms and Architectures (SPAA), pp. 286 - 294, San Diego, CA, June 2003.

[9] A. Tavory, M. Feder, and D. Ron, "Bounds on linear codes for network multicast", Electronic Colloquium on Computational Complexity (ECCC), Comment on Report 33, pp. $1-28,2003$.

[10] Y. Wu, K. Jain, and S.-Y. Kung, "A unification of network coding and tree packing (routing) theorems", IEEE Transactions on Information Theory and IEEE/ACM Transactions on Networking (joint issue), vol. 52, no. 6, pp. 2398 - 2409, June 2006

[11] R. W. Yeung, A First Course in Information Theory, Kluwer, 2002.

Note: This paper appears in the proceedings of the 44th Annual Allerton Conference on Communication, Control, and Computing, held September 27-29, 2006. 